\title{
Event-Driven Power-Law Relaxation in Weak Turbulence
}

\author{
Ludovico Silvestri, ${ }^{1}$ Leone Fronzoni, ${ }^{1}$ Paolo Grigolini, ${ }^{1,2,3}$ and Paolo Allegrini ${ }^{1}$ \\ ${ }^{1}$ Dipartimento di Fisica “E. Fermi,” Università di Pisa and INFM CRS-SOFT, Largo Pontecorvo 3, 56127 Pisa, Italy \\ ${ }^{2}$ Center for Nonlinear Science, University of North Texas, P.O. Box 311427, Denton, Texas 76203-1427, USA \\ ${ }^{3}$ Istituto dei Processi Chimico Fisici del CNR Area della Ricerca di Pisa, Via G. Moruzzi 1, 56124 Pisa, Italy
}

(Received 13 October 2008; published 5 January 2009)

\begin{abstract}
We characterize the spectral properties of weak turbulence in a liquid crystal sample driven by an external electric field, as a function of the applied voltage, and we find a $1 / f$ noise spectrum $S(f) \propto 1 / f^{\eta}$ within the whole range $0<\eta<2$. We theoretically explore the hypothesis that the system complexity is driven by non-Poisson events resetting the system through creation and annihilation of coherent structures, retaining no memory of previous history (crucial events). We study the time asymptotic regime by means of the density $\psi(\tau)$ of the time distances between two crucial events, yielding $\eta=3-\mu$, where $\mu$ is defined through the long-time form $\psi(\tau) \propto 1 / \tau^{\mu}$, with $1<\mu<3$. The system regression to equilibrium after an abrupt voltage change experimentally confirms the theory, proving violations of the ordinary linear response theory for both $\eta>1$ and $\eta<1$.
\end{abstract}

DOI: 10.1103/PhysRevLett.102.014502

Liquid crystals in the electrohydrodynamic convection (EHC) regime [1-3] afford attractive examples of spatiotemporal chaos generated by the self-organization of many interacting units [4]. Their power spectrum is

$$
S(f) \propto \frac{1}{f^{\eta}},
$$

with $0<\eta<2$, namely, the ubiquitous $1 / f$ noise. The discussion of the origin of $1 / f$ noise, even if limited to the $\mathrm{EHC}$ regime, is an issue of general interest. In fact, a general theory of $1 / f$ noise is one of the holy grails in statistical physics [5], and although a comprehensive discussion of this subject is beyond the scope of this Letter, we shall get results that will lead us to rethink the $1 / f$ noise theoretical foundation from an overlooked perspective: Some authors $[6,7]$ have studied fluctuations generated by fractal renewal processes, namely, the case where the decorrelation of a fluctuation is caused by unpredictable events and the time distance between two consecutive events is characterized by a distribution density $\psi(\tau)$ that in the time asymptotic limit is proportional to $1 / \tau^{\mu}$. These authors $[6,7]$ found

$$
\eta=3-\mu,
$$

making $S(f)$ divergent for $f \rightarrow 0$ and $\mu<3$. For this reason, we shall refer to the renewal events with $\mu<3$ as crucial events.

The main goal of this Letter is to prove the renewal origin of the EHC $1 / f$ noise. We note that the transmitted EHC light is determined by the defect creation or annihilation [8], with the defect annihilation or creation corresponding to a sudden increase (light "on") or decrease (light "off") of the light transmittivity, and, consequently, to a dichotomous signal. We further note that the experimental assessment of the spectrum of Eq. (1) establishes a
PACS numbers: 47.65. $-\mathrm{d}, 05.40 . \mathrm{Fb}, 61.30 .-\mathrm{v}, 47.52 .+\mathrm{j}$

contact with a problem of fundamental interest, in the field of statistical physics: the ergodicity breakdown. In fact, the condition $\eta>1$, under the assumption that crucial events exist, leads through Eq. (2) to $\mu<2$, and consequently to the ergodicity breakdown studied in the recent work of Margolin and Barkai [7]. The condition $\eta<1$, which is in principle compatible with a stationary correlation function proportional to $1 / t^{1-\eta}$, violates the ergodic condition established by the Lee's ergometer [9].

To realize our goal, we shall supplement the spectrum evaluation with the observation of the system's response to perturbation. We shall compare this response to a theoretical prescription, resting on the action of crucial events, which is exact under the following assumption: The abrupt onset of a control potential (see the illustration of the experimental method) resets the memory of the entire system, and makes the waiting time distribution density for the first event occurrence identical to that for the subsequent events. This common waiting time distribution density has the form

$$
\psi_{ \pm}(\tau)=(\mu-1) \frac{T_{ \pm}^{\mu-1}}{\left(\tau+T_{ \pm}\right)^{\mu}},
$$

according to whether we consider the light on state $(|+\rangle)$ or the light off state $(|-\rangle)$. The two states share the same index $\mu$ with the bias $T_{+} \neq T_{-}$.

Notice that the choice of the form of Eq. (3), going beyond the merely time asymptotic treatments of Refs. [6,7], is necessary to properly describe the perturbation influence [10]. The power-law behavior shows up in the long-time regime after a short transient that in the specific case of this Letter, as we shall see, is characterized by oscillating behavior. The amplitude of $\psi_{ \pm}$in the long time is independent of the detailed form of the transient regime. In our model, the crucial events occur at times $t_{i}$ 
and the duration of each laminar region, namely, the time interval between two consecutive crucial events, $\tau_{i}=$ $t_{i+1}-t_{i}$, is derived either from $\psi_{+}(\tau)$ or from $\psi_{-}(\tau)$, according to a fair coin tossing prescription. The signal $\xi_{S}(t)$ is generated by correspondingly assigning to each laminar region the value of 1 or -1 . We make the average over infinitely many realizations and we write $\Pi(t) \equiv$ $\left\langle\xi_{S}(t)\right\rangle=P_{+}-P_{-}$, namely, as the difference between the average population $P_{+}$in $|+\rangle$and the average population $P_{-}$in $|-\rangle$. These properties read

$$
P_{ \pm}(t)=c_{ \pm} p_{ \pm}(t)+c_{\mp}\left[1-p_{\mp}(t)\right],
$$

with $c_{ \pm}=(1 \pm k) / 2$, and where the functions $p_{ \pm}(t)$ are defined as the conditional probabilities of finding the system in the $| \pm\rangle$ state given that it was in the same state at $t=0$. Thus, $\Pi(0)=c_{+} p_{+}(0)-c_{-} p_{-}(0)=c_{+}-c_{-}=$ $k$. It is convenient to work in the Laplace representation $\hat{f}(u)=\int_{0}^{\infty} f(t) \exp (-u t) d t$. We know [10]

$$
\hat{p}_{ \pm}(u)=\frac{1-\hat{q}_{ \pm}(u)}{u\left[1-\hat{q}_{+}(u) \hat{q}_{-}(u)\right]},
$$

where $q_{+}(t)\left[q_{-}(t)\right]$ denotes the probability that an event occurring at time $t$ may generate a transition from the state $|+\rangle(|-\rangle)$ to the state $|-\rangle(|+\rangle)$. These distribution densities are related to the perturbed $\psi_{ \pm}(t)$ via

$$
\hat{q}_{ \pm}(u)=\frac{\hat{\psi}_{ \pm}(u)}{2-\hat{\psi}_{ \pm}(u)} .
$$

Note that for $u \rightarrow 0[10]$

$$
\hat{\psi}_{ \pm}(u)=1-\Gamma(2-\mu)\left(T_{ \pm} u\right)^{\mu-1}-\frac{T_{ \pm} u}{\mu-2} .
$$

Using these properties we write

$$
\Pi(t)=L(t)+O(t),
$$

where

$$
\hat{L}(u)=\hat{p}_{+}(u)-\hat{p}_{-}(u),
$$

and

$$
\hat{O}(u)=k\left[\hat{p}_{+}(u)+\hat{p}_{-}(u)-\frac{1}{u}\right] .
$$

Equations (5) and (6) plugged into (9) and (10) yield

$$
\hat{L}(u)=-\frac{1}{u} \frac{\hat{\psi}_{+}(u)-\hat{\psi}_{-}(u)}{2-\hat{\psi}_{+}(u)-\hat{\psi}_{-}(u)}
$$

and

$$
\hat{O}(u)=\frac{2 k}{u} \frac{1-\hat{\psi}_{+}(u)-\hat{\psi}_{-}(u)+\hat{\psi}_{+}(u) \hat{\psi}_{-}(u)}{2-\hat{\psi}_{+}(u)-\hat{\psi}_{-}(u)} .
$$

Finally, with the help of (7), we make the long-time prediction

$$
\begin{array}{ll}
L(t)=\frac{\Delta_{\mu}}{\Sigma_{\mu}}\left[1+\left(\frac{\Delta_{2}}{\Delta_{\mu}}-\frac{\Sigma_{2}}{\Sigma_{\mu}}\right) \frac{t^{\mu-2}}{\Gamma(\mu-1)}\right], \quad \text { if } \mu<2, \\
L(t)=\frac{\Delta_{2}}{\Sigma_{2}}\left[1+\left(\frac{\Delta_{\mu}}{\Delta_{2}}-\frac{\Sigma_{\mu}}{\Sigma_{2}}\right) \frac{t^{2-\mu}}{\Gamma(3-\mu)}\right], \quad \text { if } \mu>2,
\end{array}
$$

where $\quad \Sigma_{2}\left(\Delta_{2}\right) \equiv\left(T_{+} \pm T_{-}\right) /(\mu-2) \quad$ and $\quad \Sigma_{\mu}\left(\Delta_{\mu}\right) \equiv$ $\Gamma(2-\mu)\left(T_{+}^{\mu-1} \pm T^{\mu-1}\right)$. As to $O(t)$, we have the following theoretical prediction

$$
O(t)=k \frac{A}{t^{\mu-1}},
$$

where $A=\left[2\left(T_{+} T_{-}\right) /\left(T_{+}^{\mu-1}+T^{\mu-1}\right)\right]$ for $\mu<2$ and $A=$ $\left[\left(T_{+} T_{-}^{\mu-1}+T_{-} T_{+}^{\mu-1}\right) /\left(T_{+}+T_{-}\right)\right]$for $\mu>2$. In the limiting case of a weak perturbation, $T_{+} \rightarrow T_{-}, A \rightarrow$ $T^{\mu-1}$, where $T \equiv\left(T_{+}+T_{-}\right) / 2$, and we have an Onsager relaxation. In the same limit $T_{+} \rightarrow T_{-}$, Eqs. (13) and (14) coincide with the prediction of the event-dominated fluctuation-dissipation theorem (EDFDT) of Ref. [10].

The conventional linear response theory (LRT) [9], requiring the existence of a stationary correlation function, cannot be used in the case $\eta>1[6,7]$. However, when the stationary correlation function of $\xi_{S}(t)$ exists, but it is not integrable, namely, $\Phi_{\xi}(t) \propto 1 / t^{\beta}$, with $\beta<1$, it yields $\eta=1-\beta<1$ [5], generating ergodicity breakdown according to Lee [9], without requiring the action of crucial events. We note that in this case the LRT would yield $\left(k_{1}\right.$ and $k_{2}$ being suitable constants)

$$
\Pi(t)=k_{1}\left(1-\frac{1}{t^{\beta}}\right)+\frac{k_{2}}{t^{\beta}},
$$

in contrast with our theoretical prediction for $\mu>2$.

The experimental observation of the EHC fluctuations allows us to asses that the whole range $0<\eta<2$ is dominated by crucial events. Our setup consists in a planar-aligned nematic liquid crystal (MBBA, or methoxybenzylidene butylaniline) sandwiched between two transparent glass plates coated with indium oxide to allow electrical conductivity. We study the conducting regime by applying an AC electric potential difference between the two plates with a frequency of $60 \mathrm{~Hz}$. Sample dimensions are: Width $d=10 \pm 1 \mu \mathrm{m}$ and lateral dimensions $A=1.0 \pm 0.1 \mathrm{~cm}^{2}$, yielding aspect ratio $s=1000 \pm 110$. The sample is maintained at $18.000 \pm 0.004{ }^{\circ} \mathrm{C}$ temperature. This precision is achieved using a double thermostat, where a high-precision resistance thermistor has the role of warming up the sample, cooled down by a thermal bath at a lower temperature $\left(8.00 \pm 0.01{ }^{\circ} \mathrm{C}\right.$ in our setup), using a second thermostat. The lamp under the sample is stabilized through a photodiode-driven feedback circuit. A CCD captures the sample images magnified by a polarizer microscope and sends it to a personal computer equipped with 
a digital video-acquisition card that transforms digital images into 8-bit grayscale numerical matrices for subsequent offline analysis.

Above a threshold voltage $V_{0} \approx 20 \mathrm{~V}$, a convective instability emerges, giving rise to the so-called Williams domains [3], visualized as a regular pattern of light vs. darkness stripes. As the voltage is further increased, the defects start to appear in the regular pattern with a specific dynamical behavior: It is possible to see births, deaths, drifts, and oscillations of defects through the observed region. A review of this phenomenon can be found in [2]. The control parameter $\epsilon \equiv V / V_{0}-1$, called reduced voltage, is defined in such a way that $\epsilon>0$ corresponds to the region where these dynamics occur. The time scales (inverses of drift velocities, oscillation frequencies, and death-birth rates) decrease with increasing $\epsilon$, visually showing a less spacially-correlated turbulent pattern. For $0<\epsilon<\epsilon_{1}=0.21 \pm 0.01$ we have spacial inhomogeneity in the pattern and the system does not equilibrate. We see lines of defects separating different zones of regular stripes with different orientations. Further increasing $\epsilon$, these regular domains become smaller and shorter living, till $\epsilon=$ $\epsilon_{1}$, which signals the transition to another regime, $\epsilon_{1}<$ $\epsilon<\epsilon_{2}$ or dynamic scattering mode 1 [2], where inhomogeneity disappears, and a stationary correlation function $\Phi_{\xi}(t)$ can be defined. The transition to fully developed turbulence ( $\epsilon>\epsilon_{2}$, or dynamic scattering mode 2 [2]) is beyond the scope of this study, focusing on the presence of $1 / f$ noise, which disappears at $\epsilon=0.9 \pm 0.1<\epsilon_{2}$. Above this point the spectral behavior lacks any power-law form, for $f \rightarrow 0$.

We note that the signal $\xi_{S}(t)$ of the earlier illustrated theory is the global luminosity which is the sum of individual contributions, whose intensity decreases or in-

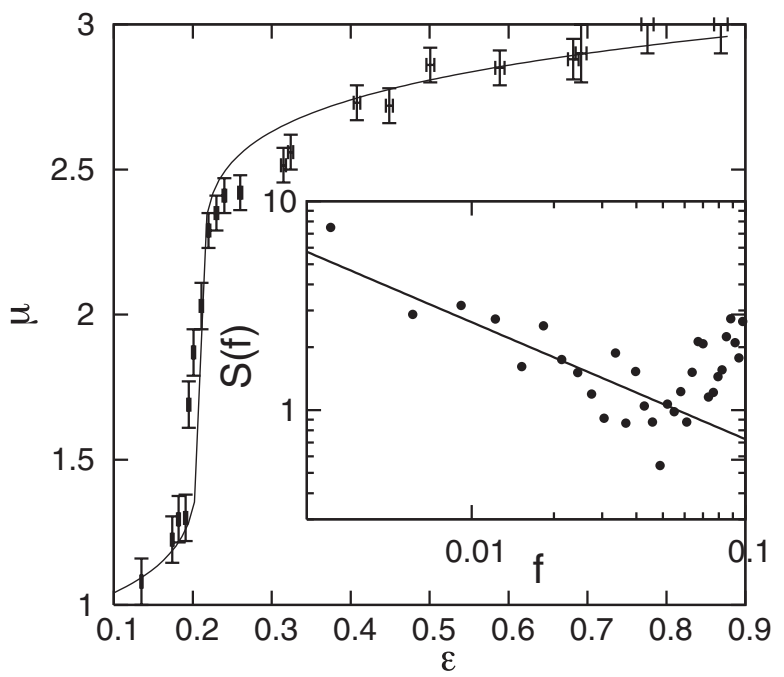

FIG. 1. Points denote $\mu$ as a function of the reduced voltage $\epsilon$; solid line is only a guide to the eye. Inset: Points denote power spectrum for $\epsilon=0.260 \pm 0.003$; line is a fit yielding $\mu=2.44$. creases with the creation or annihilation of defects. Although the cooperative nature of the process is expected to generate a global dichotomous signal according to the theory of Ref. [11], see [12], the single $\xi_{S}(t)$ turns out to be erratic, thereby making it convenient to make averages over different realizations of $\xi_{S}(t)$, corresponding to the same abrupt change of $\epsilon$. According to the theory this average has to be identified with $\Pi(t)$.

In Fig. 1, we show the indexes $\mu$ stemming from the $1 / f^{\eta}$ spectra through Eq. (2). Spectral analysis was performed on recorded 1-h-length signals of unperturbed $\Pi(t)$ 's, with averages on moving windows of duration $327.68 \mathrm{~s}$, corresponding to $2^{13}$ points at $0.04 \mathrm{~s}$ sampling time, overlapping for half of the window lengths. We also evaluated $\mu$ using other independent techniques, like the diffusion entropy [13] and the detrended fluctuation analysis [14] giving the same results with greater accuracy. In Fig. 1 error bars are reported according to this higher precision, but the discussion of diffusion entropy and detrended fluctuation analysis will be omitted here. We note that $\mu$ as a function of $\epsilon$ shows a fast change around $\mu=2$, signaling the transition from the nonergodic regime of [7] to the nonergodic regime of [9]. In the inset of Fig. 1, we show how the resulting measure is consistent with the empirical power spectrum.

There is no overwhelming evidence up to this stage that the $1 / f$ complexity is due to renewal events. To reinforce our conviction that crucial events lead the dynamics, we use our experimental setup to prove that the renewal theory, adopted to derive Eqs. (8) and (13)-(15), affords a satisfactory picture of the system's regression to equilibrium after an abrupt change of $\epsilon$, in accordance with the

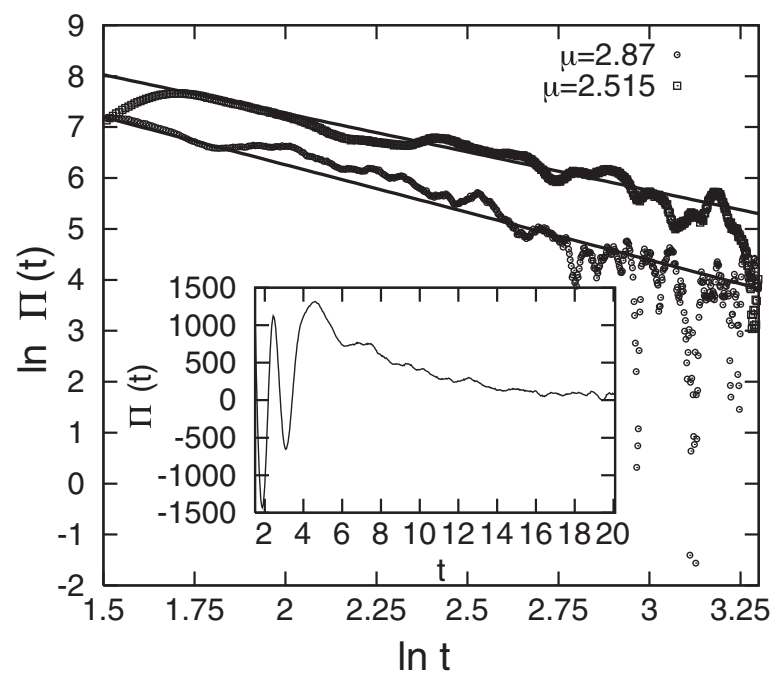

FIG. 2. Relaxation of $\Pi(t)$ for $\mu=2.515$ and $\mu=2.87$ (upper and lower set of points and curves, respectively). Points represent experimental observations; solid lines represent the theoretical power-index relaxation $1 / t^{\mu-1}$. Inset: Same in natural time scale for $\mu=2.87$, showing some oscillations before the inverse-power-law decay. 


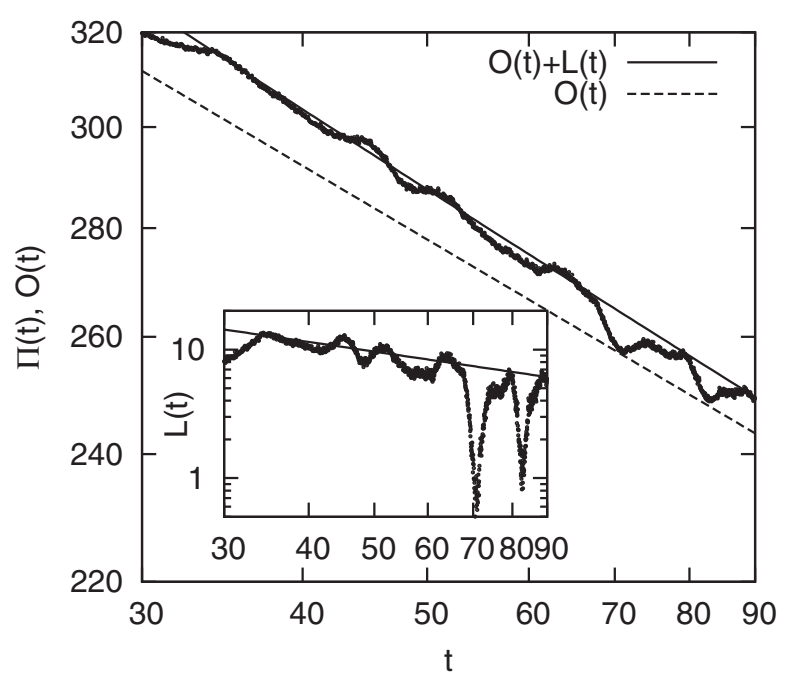

FIG. 3. Relaxation of $\Pi(t)$ for $\mu=1.225$. Points represent experimental $\Pi(t)$. The solid line represent the theoretical sum of two power-index relaxations, $L(t) \propto 1 / t^{2-\mu}$ and $O(t) \propto$ $1 / t^{\mu-1}$; the dashed line represents relaxation $O(t)$ only. Inset: Points denote experimental $L(t)=\Pi(t)-O(t)$ (from main panel), solid line represents an inverse-power law $\propto 1 / t^{2-\mu}$.

EDFDT of [10] and in conflict with the ordinary LRT. For this purpose, we modulate the voltage amplitude with a square wave of period $P=100 \mathrm{~s}$. At the beginning the system is below threshold for a period $P_{r}=10 \mathrm{~s}$ in order to quench the system and delete any memory of spacial correlation. Then for a period $P_{\epsilon}=P-P_{r}=90 \mathrm{~s}$ the system is left to reach its stationary light transmittivity. Averages were performed on 200 periods to establish a connection with a Gibbs-like averages, unavoidable in the nonergodic regime.

Let us move now to illustrate the important results of the perturbation experiment, for both $\mu>2$ and $\mu<2$. In both cases, as earlier mentioned, we make an average over many realizations, thereby generating $\Pi(t)$, a quantity related to the transmitted light intensity, in arbitrary units: $\Pi(t)$ is a linear function of this luminosity, chosen in a way that it decays to zero (corresponding to the stationary luminosity value) from a positive value.

The case $\mu>2$ is illustrated in Fig. 2, with the two cases $\epsilon=0.324(\mu=2.515)$ and $\epsilon=0.513(\mu=2.87)$. In both cases we compare the experimental results to the theoretical prediction, $O(t)$ of Eq. (15), namely, a relaxation decay proportional to $1 / t^{\mu-1}$. We see that the data follow the theoretical prediction with a good agreement. Notice that the agreement is asymptotic, and that the regression starts following an inverse-power law after a few oscillations, as shown in the inset of Fig. 2. The physical reason of these oscillations is the fast relaxation of the background pattern transmittivity, which is superimposed to the defects dynamics.

The case $\mu=1.225(<2)$ is shown in Fig. 3, where the full line corresponds to the theoretical prediction (8). The dominant factor $O(t)$ of Eq. (15) can be subtracted to the experimental relaxation, and compared to the dynamical contribution $L(t)$ of Eq. (13). As shown in the insert of Fig. 3, this procedure unravels a contribution to relaxation proportional to $1 / t^{2-\mu}$. Notice that a sequence of crucial events with $\mu<2$ makes the Kolmogorov-Sinai entropy [15] increase as $L^{\mu-1}$, generating a randomness rate per unit of time decreasing as $1 / L^{2-\mu}$. This shows that the EHC fluctuations, requiring the EDFDT of Ref. [10], are a manifestation of this weakly chaotic condition.

In conclusion, this Letter proves that the $1 / f$ noise in the EHC regime is generated by crucial events, and that the ideal $1 / f$ noise, $\eta=1$, namely, $\mu=2$, is the boundary between the nonergodic regime studied by the authors of Ref. [7] $(\mu<2)$ and a regime that although is in principle compatible with the stationary assumption would make the Lee's ergometer declare ergodicity breakdown. We expect that this result will force the researchers in the field of nonequilibrium statistical physics to rethink the $1 / f$ noise origin as a manifestation of crucial events.

P. G. thanks ARO and Welch for financial support of this work through Grants No. W911NF-08-1-0177 and No. B1577 , respectively.

[1] J. T. Gleeson, Phys. Rev. E 63, 026306 (2001); D. R. Spiegel, E. R. Johnson, and S. R. Saucedo, Phys. Rev. E 73, 036317 (2006); T. Tóth-Katona and J. T. Gleeson, Phys. Rev. Lett. 91, 264501 (2003).

[2] T. Tóth-Katona and J. T. Gleeson, Phys. Rev. E 69, 016302 (2004).

[3] S. Kai, N. Chizumi, and M. Kohno, Phys. Rev. A 40, 6554 (1989).

[4] S. T. Bramwell, P.C. W. Holdsworth, and J.-P. Pinton, Nature (London) 396, 552 (1998); S. T. Bramwell et al., Phys. Rev. Lett. 84, 3744 (2000).

[5] R. Voss and J. Clarke, Phys. Rev. Lett. 36, 42 (1976).

[6] S. B. Lowen and M.C. Teich, Phys. Rev. E 47, 992 (1993).

[7] G. Margolin and E. Barkai, J. Stat. Phys. 122, 137 (2006).

[8] T. Tóth-Katona, J. R. Cressman, W. I. Goldburg, and J. T. Gleeson, Phys. Rev. E 68, 030101(R) (2003).

[9] M. H. Lee, Phys. Rev. Lett. 98, 110403 (2007); Acta Phys. Pol. B 39, 1035 (2008).

[10] P. Allegrini, M. Bologna, P. Grigolini, and B. J. West, Phys. Rev. Lett. 99, 010603 (2007).

[11] S. Bianco, E. Geneston, P. Grigolini, and M. Ignaccolo, Physica (Amsterdam) 387A, 1387 (2008).

[12] Movies of the defect's dynamics are available at http:// mail.df.unipi.it/ allegrip/liquid_crystal.html.

[13] N. Scafetta, P. Hamilton, and P. Grigolini, Fractals 9, 193 (2001); P. Grigolini, L. Palatella, and G. Raffaelli, Fractals 9, 439 (2001).

[14] C. K. Peng et al., Phys. Rev. E 49, 1685 (1994).

[15] P. Gaspard and X.-J. Wang, Proc. Natl. Acad. Sci. U.S.A. 85, 4591 (1988). 